Radiologe 2010 · 50:588

DOI 10.1007/s00117-010-2026-5

Online publiziert: 3. Juli 2010

(c) Springer-Verlag 2010

\author{
C. Loewe \\ Klinische Abteilung für Kardiovaskuläre und Interventionelle Radiologie, \\ Universitätsklinik für Radiodiagnostik Wien
}

\title{
Motto: Jeder, der kann, soll auch Betablocker erhalten!
}

Folgender Originalbeitrag wurde für Sie gelesen und kommentiert

Graaf FR de, Schuijf JD, Velzen JE van et al (2010) Evaluation of contraindications and efficacy of oral beta blockade before computed tomographic coronary angiography. Am J Cardiol 105:767-772

Nicht dass wir uns das nicht schon immer gedacht hätten (nachher ist man bekanntlich immer klüger!) - aber nun ist es amtlich und schwarz auf weiß nachzulesen: auch in Zeiten der immer besser werdenden zeitlichen Auflösung in der Computertomographie der Koronararterien - egal ob nun diese verbesserte Zeitauflösung der jeweiligen Firmenphilosophie entsprechend Doppelröhrensystemen, extrem breiten Detektoren oder der Kombination von beiden zuzuschreiben ist - brauchen wir für schöne Bilder Betablocker wie einen Bissen Brot. Wenngleich Gerätehersteller ihr jeweils neuestes Spitzen-CT-Gerät auch damit anpreisen, dass nunmehr Betablocker nicht notwendig wären, ist doch wohltuend, nunmehr dank Fleur de Graaf einen hervorragenden Artikel in Händen zu halten, der das Gegenteil beweist und Marketingaussagen als solche entlarvt. Die Aussage der Arbeit ist diesbezüglich klar und eindeutig: auch wenn die Datenakquisition während eines einzigen Herzschlags erfolgt, ist eine Reduktion der Herzfrequenz essenziell für optimale Bildqualität und reduzierte Strahlenexposition.

\section{Aber alles schön der Reihe nach}

In der kürzlich publizierten prospektiven Studie wurden mehr als 500 PatientInnen evaluiert, die zum Herz-CT zugewiesen worden waren - aus welcher Fragestellung heraus auch immer. Das Studiendesign war denkbar einfach und klar: die PatientInnen wurden entsprechend ihrer Basisherzfrequenz und ihrer etwaigen Kontraindikationen gegen die Gabe von Betablockern in entsprechende Gruppen eingeteilt. Und siehe da: die Gruppe der PatientInnen mit Kontraindikationen gegen eine Betablockergabe bzw. eine ausreichend hohe Betablockergabe hatten eine relevant schlechtere Bildqualität in der Herz-CT als die PatientInnen mit einer niedrigen Herzfrequenz oder einer ausreichend hohen Betablockade. Aber das ist nicht die einzige wichtig Aussage der vorliegenden Studie: zusätzlich zur Auswertung der Bildqualität wurde erstmalig in einer großen Studie auch die tatsächliche Häufigkeit von Kontraindikationen gegen eine Betablockergabe bzw. gegen eine ausreichend hohe Gabe von Betablockern ermittelt - und auch dieses Ergebnis ist durchaus interessant und überraschend: Bei immerhin 16\% der evaluierten Patienten bestanden Kontraindikationen (Asthma, AV-Block, schwere Aortenstenose, schwere systolische Dysfunktion, Allergie gegen Metropolol). Und von diesen $16 \%$ (also etwa 90 PatientInnen) wurde die Zielherzfrequenz von 65 oder darunter in 60\% nicht erreicht - mit entsprechenden Konsequenzen.
Also: Jeder, der kann (also keine Kontraindikationen gegen Betablocker hat!) und es braucht (also eine Herzfrequenz über 65,) soll auch Betablocker bekommen. Unabhängig vom Scanner, denn schließlich wurde ein Teil der PatientInnen an einem 32-Zeiler untersucht, der andere Teil an einem 64 -Zeiler - ohne relevanten Unterschied.

Einzige Limitation der Studie ist aus meiner Sicht die Tatsache, dass ausschließlich orale Betablocker in dieser Studie evaluiert wurden, wobei die zentrale Aussage - Betablocker sollen immer gegeben werden - dadurch nicht wesentlich beeinflusst wird.

Zusammenfassend also eine sehr praxisnahe und praxisrelevante Arbeit, die fern aller Marketingaussagen die derzeitige klinische Realität des Herz-CT evaluiert und uns eindrücklich die Notwendigkeit der Herzfrequenzbeeinflussung - auch in Zeiten der ultraschnellen CTScanner - vor Augen führt.

\section{Korrespondenzadresse}

Prof. Dr. C. Loewe

Klinische Abteilung für Kardiovaskuläre und Interventionelle Radiologie, Universitätsklinik für Radiodiagnostik Wien, Währinger Gürtel 18-20, A-1090 Wien, Österreich

christian.loewe@meduniwien.ac.at 